\title{
Association of IKZF1 SNPs in cold medicine-related Stevens-Johnson syndrome in Thailand
}

\author{
Patchima Chantaren ${ }^{1 \dagger}$, Passara Jongkhajornpong ${ }^{2 \dagger}$, Mayumi Ueta $^{3^{*}+}$ (1), Vilavun Puangsricharern ${ }^{1}$, \\ Kaevalin Lekhanont ${ }^{2}$, Phattrawan Pisuchpen ${ }^{1}$, Pinnita Prabhasawat ${ }^{4}$, Kanya Suphapeetiporn ${ }^{5}$ \\ and Shigeru Kinoshita ${ }^{3}$
}

\begin{abstract}
Purpose: Our meta-analysis of several ethnic groups (Japanese, Korean, Indian, Brazilian) revealed a significant genome-wide association between cold medicine-related SJS/TEN (CM-SJS/TEN) with severe ocular complications (SOC) and IKZFI SNPS, suggesting that IKZFI might be a potential marker for susceptibility to CM-SJS/TEN with SOC. In this study, we examined the association between CM-SJS/TEN with SOC and the IKZF1 SNPs in the Thai population.

Methods: 57 CM-SJS/TEN with SOC and 171 control samples were collected at Chulalongkorn University and Mahidol University. Genomic DNA samples were genotyped for the IKZF1 SNPs at Kyoto Prefectural University of Medicine in Japan using the TaqMan SNP genotyping assay.

Results: The four SNPs previously reported to be associated with CM-SJS/TEN with SOC in the Japanese were examined in the Thai samples. Although the number of Thai cases $(n=57)$ was small, a significant association between CM-SJS/TEN with SOC and IKZFI SNPs which included rs4917014 (T VS G, OR=2.9, $p=0.0012, P C=0.0049$ ), rs 4917129 ( $T$ vs $C, O R=2.8, p=0.0026, P C=0.010$ ) and $r s 10276619$ ( $G$ vs $A, O R=1.8, p=0.012, P C=0.048$ ) was identified.

Conclusion: In addition to the Japanese, Korean and Indian populations, Thai cases with CM-SJS/TEN and SOC were significantly associated with IKZF1 SNPS. With our previous report of the critical role of IKZF1 in mucocutaneous inflammation, these results suggest that IKZFI is important in the pathogenesis of CM-SJS/TEN with SOC.
\end{abstract}

Keywords: Stevens-Johnson syndrome, Cold medicine, Severe ocular complications, SNPS, IKZF1

To the editor

Stevens-Johnson syndrome (SJS) and its severe type, toxic epidermal necrolysis (TEN), are acute inflammatory vesiculobullous reactions of the skin and mucosa including the ocular surface, oral cavity, and genitals. Severe ocular complications (SOC) appear in about half of SJS/TEN patients diagnosed by dermatologists [1]. Cold medicines $(\mathrm{CM})$, including multi-ingredient cold

\footnotetext{
*Correspondence: mueta@koto.kpu-m.ac.jp

†Patchima Chantaren, Passara Jongkhajornpong and Mayumi Ueta contributed equally as first authors

${ }^{3}$ Department of Frontier Medical Science and Technology for Ophthalmology, Kyoto Prefectural University of Medicine, 465 Kajiicho,

Hirokoji, Kawaramachi, Kamigyoku, Kyoto 602-0841, Japan

Full list of author information is available at the end of the article
}

medications and non-steroid anti-inflammatory drugs (NSAIDs) were the main causative drugs of SJS/TEN with SOC [2]. In the acute stage, in addition to skin eruption and erosion, SJS/TEN with SOC patients manifest severe conjunctivitis with corneal and conjunctival erosion and pseudo-membranes. Despite healing of the skin lesions, in the chronic stage of SJS/TEN with SOC, ocular surface sequelae such as severe dry eye, symblepharon, trichiasis, scaring of palpebra conjunctiva, and conjunctival invasion into the cornea may persist [3]. While the reported annual incidence of SJS/TEN is very rare (only $1-6 / 10^{6}$ individuals), its mortality rate is high ( $3 \%$ for SJS and $27 \%$ for TEN) [4]. We previously reported that the IKZF1 gene was strongly associated with CM-SJS/TEN with SOC in Japanese patients [5]. In addition, a meta-analysis 
of several ethnic groups (Japanese, Korean, Indian, Brazilian) revealed a significant genome-wide association between CM-SJS/TEN with SOC and IKZF1, suggesting that $I K Z F 1$ might be a potential marker for susceptibility to CM-SJS/TEN with SOC [5]. In this study, we examined the association between Thai CM-SJS/TEN with SOC and the IKZF1 SNPs, known to be associated with the Japanese CM-SJS/TEN with SOC.

The CM-SJS/TEN with SOC and control samples were collected at Chulalongkorn University (King Chulalongkorn Memorial Hospital) and Mahidol University (Ramathibodi Hospital and Siriraj Hospital). Genomic DNA samples were genotyped for the IKZF1 SNPs at Kyoto Prefectural University of Medicine in Japan. The study was approved by the institutional review board of all institutes. Protocol explanation and obtaining written informed consent were done in all participants before starting experimental procedures. All experimental processes were complied with the principles set forth in the Helsinki declaration. The diagnostic criteria of SJS/ TEN were based on a confirmed history of acute onset of high fever, skin eruption with at least two sites of serious mucocutaneous involvement including the oral mucosa and the ocular surface [5]. Thai healthy volunteers were used as controls. CM was defined as the drug that patients took for relieving cold symptoms including nonsteroidal anti-inflammatory drugs (NSAIDS), acetaminophen, and other multi-ingredient cold medications [6]. We previously reported that in Japanese, for SIS/TEN with SOC, acetaminophen was a main drug of CM; $48 \%$ of the patients have taken acetaminophen before developing SJS/TEN with SOC [6]. In Thailand, paracetamol (which is equal to acetaminophen) might be also important causative drug of CM, because 20 of 57 (35\%) patients have taken paracetamol before developing it. The patients were classified as having SOC if the following manifestations were detected; severe conjunctivitis, pseudomembrane, and epithelial defect on the ocular surface in the acute stage and/or ocular sequelae such as dry eye, trichiasis, symblepharon, and conjunctival invasion into the cornea in the chronic stage [6].

Of 57 CM-SJS/TEN with SOC, 23 were male and 34 were female; their age ranged from 6 to 73 years [median $42.3 \pm 15.6(\mathrm{SD})$ years]. The age at SJS/TEN onset ranged from 2 to 54 years (median $24.8 \pm 13.8$ years). The controls were 85 males and 86 females; their median age was $39.5 \pm 14.3$ years. Some of the CM-SJS/TEN patients and some of the controls had been included in our earlier studies [7].

Subjects were obtained DNA extraction from whole peripheral blood using the PAX gene blood DNA kits (Qiagen, Hilden, Germany) or from saliva using Oragene DNA (Kyodou International, Kanagawa, Japan).
The TaqMan SNP genotyping assay (Applied Biosystems, Foster City, CA) was used for the genotypes of the IKZF1 gene as previously reported [5]. Chi squared test was applied to a two-by-two contingency table for the allele frequency and the dominant and recessive models.

The 4 SNPs previously reported to be associated with CM-SJS/TEN with SOC in the Japanese were examined in the Thai samples. Although the number of Thai cases $(\mathrm{n}=57)$ was small, we again found a significant association between CM-SJS/TEN with SOC and 3 IKZF1 SNPs which included rs4917014 ( $\mathrm{T}$ vs $\mathrm{G}, \mathrm{OR}=2.9, \mathrm{p}=0.0012$, $\mathrm{Pc}=0.0049$ ), rs4917129 ( $\mathrm{T}$ vs $\mathrm{C}, \mathrm{OR}=2.8, \mathrm{p}=0.0026$, $\mathrm{Pc}=0.010)$ and $\mathrm{rs} 10276619$ ( $\mathrm{G}$ vs $\mathrm{A}, \mathrm{OR}=1.8, \mathrm{p}=0.012$, Pc $=0.048)($ Table 1). Our previous results of meta-analysis in the Japanese, Korean, Indian and Brazilian showed the significant associations in 3 IKZF1 SNPs [rs4917014 ( $\mathrm{T}$ vs $\mathrm{G}, \mathrm{OR}=2, \mathrm{p}=8.5 \times 10^{-11}$ ) (which is equal to $(\mathrm{G}$ (minor allele) vs $\mathrm{T}$ (major allele), $\mathrm{OR}=0.5$ ) in the previous paper), rs4917129 ( $\mathrm{T}$ vs $\mathrm{C}, \mathrm{OR}=2, \mathrm{p}=8.0 \times 10^{-9}$ ) (which is equal to (C (minor allele) vs $\mathrm{T}$ (major allele), $\mathrm{OR}=0.5$ in the previous paper)) and rs10276619 (G vs A, $\left.\mathrm{OR}=1.8, \mathrm{p}=4.3 \times 10^{-9}\right)$ ] [5]. Present results in the Thai population are in concordance with our previous report.

Our functional analysis of SNPs of the IKZFI gene revealed that the ratio of the splicing isoforms Ik2/Ik1 could be affected by $I K Z F 1$ SNPs significantly associated with susceptibility to CM-SJS/TEN with SOC [5]. The quantity of the Ik2 isoform is increased in disease-protective genotypes of IKZF1 (rs4917014 G/G and rs10276619 A/A) [5]. As Ikaros 2, an Ik2 isoform lacks the DNAbinding ability and seems to be dominant-negative. It is possible that the function of Ikaros, the protein of IKZFI, is enhanced in CM-SJS/TEN with SOC [5].

Ikaros is a transcription factor that regulates numerous biological events. It was reported that Ikaros-null mice lack B-lineage cells, NK cells, peripheral lymph node- and fetal T-cells, thus Ikaros family members regulate important cell-fate decisions in the development of the adaptive immune system [8]. On the other hand, we have reported that epithelium might be contribute to the pathobiology of CM-SJS/TEN with SOC [9]. Thus, we produced K5-Ikzf1-EGFP transgenic mice (Ikzf1 $\mathrm{Tg}$ ) by introducing the Ik1 isoform into cells expressing keratin 5, which is expressed in epithelial tissues such as the epidermis and conjunctiva and found that mucocutaneous inflammation was exacerbated in Ikzf1-Tg mice. They developed dermatitis with some having blepharoconjunctivitis [10]. Histological analysis showed not only dermatitis but also tissue inflammation in the blepharoconjunctiva, tongue, and paronychia [10], similar to the findings in patients in the acute state of SJS/TEN with SOC [9]. Our studies demonstrated that IKZF1 could play a critical role in 
Table 1 Woolf's correction

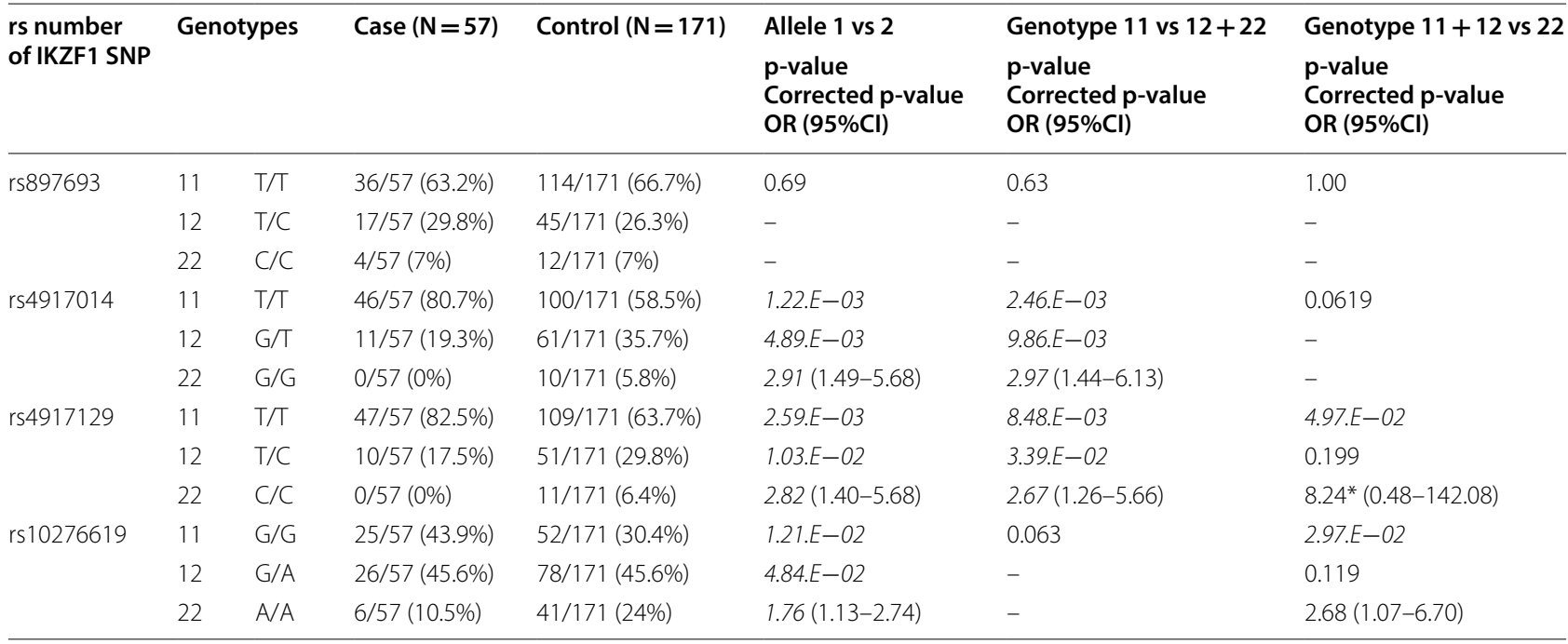

maintaining mucocutaneous homeostasis [10] and suggested that it might be implicated in the aggravation of mucocutaneous inflammation seen in the presence of CM-SJS/TEN with SOC [10].

In addition to the Japanese, Korean and Indian in our previous report [5], CM-SJS/TEN with SOC was significantly associated with IKZF1 SNPs in the Thai cases. With our previous report of the critical role of $I K Z F 1$ in mucocutaneous inflammation [8], these results suggest that IKZF1 is important in the pathogenesis of CM-SJS/ TEN with SOC.

\section{Acknowledgements}

We thank all the patients and volunteers who enrolled in our study. We also thank Ms. Hiromi Nishigaki for technical assistance.

\section{Authors' contributions}

MU made the experimental design, analyze the SNPS, wrote the text, and prepared the table. PC, PJ, PP and PP collected samples. PC, PJ, VP, KL, KS and SK contributed to the research and reviewed the manuscript. All authors read and approved the final manuscript.

\section{Funding}

This work was supported by grants-in-aid from the Ministry of Education, Culture, Sports, Science and Technology of the Japanese government, and by the JSPS Core-to-Core Program, A. Advanced Research Networks and also partly supported by grants-in-aids for scientific research from the Japanese Ministry of Health, Labor and Welfare.

\section{Ethics approval and consent to participate}

The study was approved by the institutional review board of Chulalongkorn University (King Chulalongkorn Memorial Hospital), Mahidol University (Ramathibodi Hospital and Siriraj Hospital) and Kyoto Prefectural University of Medicine.

\section{Consent for publication}

Protocol explanation and obtaining written informed consent were done in all participants before starting experimental procedures.

\section{Competing interests}

The authors declare that they have no competing interests.

\section{Author details}

${ }^{1}$ Department of Ophthalmology, Faculty of Medicine, Chulalongkorn University and Excellence Center for Cornea and Limbal Stem Cell Transplantation, King Chulalongkorn Memorial Hospital, Thai Red Cross Society, Bangkok, Thailand. ${ }^{2}$ Department of Ophthalmology, Faculty of Medicine, Ramathibodi Hospital, Mahidol University, Bangkok, Thailand. ${ }^{3}$ Department of Frontier Medical Science and Technology for Ophthalmology, Kyoto Prefectural University of Medicine, 465 Kajiicho, Hirokoji, Kawaramachi, Kamigyoku, Kyoto 602-0841, Japan. ${ }^{4}$ Department of Ophthalmology, Faculty of Medicine, Siriraj Hospital, Mahidol University, Bangkok, Thailand. ${ }^{5}$ Department of Pediatrics, Faculty of Medicine, Chulalongkorn University and Excellence Center for Medical Genetics, King Chulalongkorn Memorial Hospital, the Thai Red Cross Society, Bangkok, Thailand.

Received: 20 June 2019 Accepted: 13 November 2019

Published online: 22 November 2019

\section{References}

1. Sotozono C, Ueta M, Nakatani E, Kitami A, Watanabe H, Sueki H, lijima M, Aihara M, Ikezawa Z, Aihara Y, et al. Predictive factors associated with acute ocular involvement in Stevens-Johnson syndrome and toxic epidermal necrolysis. Am J Ophthalmol. 2015;160(2):228-37.

2. Ueta M. Results of detailed investigations into Stevens-Johnson syndrome with severe ocular complications. Invest Ophthalmol Vis Sci. 2018;59(14):DES183-91.

3. Sotozono C, Ang LP, Koizumi N, Higashihara H, Ueta M, Inatomi T, Yokoi N, Kaido M, Dogru M, Shimazaki J, et al. New grading system for the evaluation of chronic ocular manifestations in patients with Stevens-Johnson syndrome. Ophthalmology. 2007;114(7):1294-302.

4. Power WJ, Ghoraishi M, Merayo-Lloves J, Neves RA, Foster CS. Analysis of the acute ophthalmic manifestations of the erythema multiforme/Stevens-Johnson syndrome/toxic epidermal necrolysis disease spectrum. Ophthalmology. 1995;102(11):1669-76.

5. Ueta M, Sawai H, Sotozono C, Hitomi Y, Kaniwa N, Kim MK, Seo KY, Yoon KC, Joo CK, Kannabiran C, et al. IKZF1, a new susceptibility gene for cold medicine-related Stevens-Johnson syndrome/toxic epidermal necrolysis with severe mucosal involvement. J Allergy Clin Immunol. 2015;135(6):1538-45.

6. Ueta M, Kaniwa N, Sotozono C, Tokunaga K, Saito Y, Sawai H, Miyadera $H$, Sugiyama E, Maekawa K, Nakamura R, et al. Independent strong association of $\mathrm{HLA}-\mathrm{A}^{*} 02: 06$ and $\mathrm{HLA}-\mathrm{B}^{*} 44: 03$ with cold medicine-related 
Stevens-Johnson syndrome with severe mucosal involvement. Sci Rep. 2014;4:4862.

7. Jongkhajornpong P, Lekhanont K, Pisuchpen P, Chantaren P, Puangsricharern V, Prabhasawat P, Suphapeetiporn K, Kinoshita S, Ueta M. Association between HLA-B*44:03-HLA-C*07:01 haplotype and cold medicinerelated Stevens-Johnson syndrome with severe ocular complications in Thailand. Br J Ophthalmol. 2018;102(9):1303-7.

8. John $L B$, Ward $A C$. The Ikaros gene family: transcriptional regulators of hematopoiesis and immunity. Mol Immunol. 2011;48(9-10):1272-8.

9. Ueta M, Kinoshita S. Ocular surface inflammation is regulated by innate immunity. Prog Retin Eye Res. 2012;31(6):551-75.
10. Ueta M, Hamuro J, Nishigaki H, Nakamura N, Shinomiya K, Mizushima K, Hitomi Y, Tamagawa-Mineoka R, Yokoi N, Naito Y, et al. Mucocutaneous inflammation in the Ikaros family zinc finger 1-keratin 5-specific transgenic mice. Allergy. 2018;73(2):395-404.

\section{Publisher's Note}

Springer Nature remains neutral with regard to jurisdictional claims in published maps and institutional affiliations.
Ready to submit your research? Choose BMC and benefit from:

- fast, convenient online submission

- thorough peer review by experienced researchers in your field

- rapid publication on acceptance

- support for research data, including large and complex data types

- gold Open Access which fosters wider collaboration and increased citations

- maximum visibility for your research: over $100 \mathrm{M}$ website views per year

At BMC, research is always in progress.

Learn more biomedcentral.com/submissions 